\title{
SYMPTOMS OF INFECTION REFERRED TO AS HIVIAIDS
}

\section{${ }^{1}$ Md. Abu Salek, ${ }^{2}$ Jannatun Nayeem}

${ }^{1}$ Department of Mathematics, Bhawal Bodre Alam Government College,

${ }^{2}$ Department of Arts and Science

\section{ABSTRACT}

Human immunodeficiency virus infection and acquired immune deficiency syndrome (HIV/AIDS) is a spectrum of conditions caused by infection with the human immunodeficiency virus (HIV). Following initial infection, a person may not notice any symptoms or may experience a brief period of influenza-like illness. Typically, this is followed by a prolonged period with no symptoms. As the infection progresses, it interferes more with the immune system, increasing the risk of common infections like tuberculosis, as well as other opportunistic infections, and tumors that rarely affect people who have working immune systems. These late symptoms of infection are referred to as AIDS. This stage is often also associated with weight loss. HIV is spread primarily by unprotected sex (including anal and oral sex), contaminated blood transfusions, hypodermic needles, and from mother to child during pregnancy, delivery, or breastfeeding. Some bodily fluids, such as saliva and tears, do not transmit HIV.

Keywords: Symptoms of Infection, HIVIAIDS
${ }^{*}$ Correspondence to Author: Md. Abu Salek

Department of Mathematics, Bhawal Bodre Alam Government College How to cite this article: Md. Abu Salek and Jannatun Nayeem. SYMPTOMS OF INFECTION REFERRED TO AS HIVIAIDS. American Journal of Educational Research and Reviews, 2018,3:14.

\section{esci} eSciPub LLC, Houston, TX USA. Website: http://escipub.com/ 
1. Introduction: Methods of prevention include safe sex, needle-exchange programs, treating those who are infected, and male circumcision. ${ }^{[1]}$ Disease in a baby can often be prevented by giving both the mother and child antiretroviral medication. ${ }^{[8]}$ There is no cure or vaccine; however, antiretroviral treatment can slow the course of the disease and may lead to a near-normal life expectancy. ${ }^{[0] 12]}$ Treatment is recommended as soon as the diagnosis is made. Without treatment, the average survival time after infection is 11 years. ${ }^{[3]}$ With regard to unprotected heterosexual contacts, estimates of the risk of HIV transmission per sexual act appear to be four to ten times higher in lowincome countries than in high-income countries.
In low-income countries, the risk of female-tomale transmission is estimated as $0.38 \%$ per act, and of male-to-female transmission as $0.30 \%$ per act; the equivalent estimates for high-income countries are $0.04 \%$ per act for female-to-male transmission, and $0.08 \%$ per act for male-to-female transmission. The risk of transmission from anal intercourse is especially high, estimated as $1.4-1.7 \%$ per act in both heterosexual and homosexual contacts. [4] While the risk of transmission from oral sex is relatively low, it is still present.

1.1 Signs and symptoms of HIV/AIDS: There are three main stages of HIV infection. acute infection, clinical latency and AIDS. ${ }^{[2]}$

\section{Acute infection}

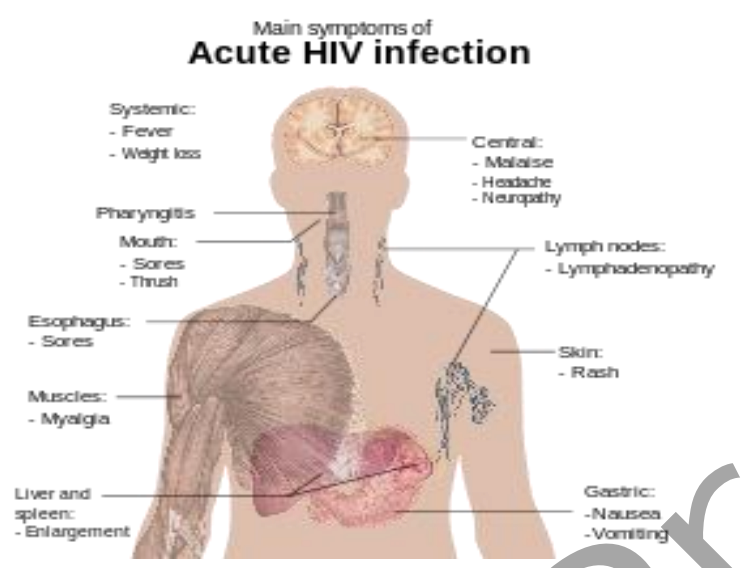

2.Main symptoms of acute HIV infection: The initial period following the contraction of HIV is called acute HIV, primary HIV or acute retroviral syndrome. ${ }^{[3]}$ Many individuals develop an influenza-like illness or a mononucleosis-like illness 2-4 weeks post exposure while others have no significant symptoms. Symptoms occur in $40-90 \%$ of cases and most commonly include fever, large tender lymph nodes, throat inflammation, a rash, headache, and/or sores of the mouth and genitals. The rash, which occurs in $20-50 \%$ of cases, presents itself on the trunk and is maculopapular, classically. Some people also develop opportunistic infections at this stage. Gastrointestinal symptoms such as nausea, vomiting or diarrhea may occur, as may neurological symptoms of peripheral neuropathy or Guillain-Barre syndrome. The duration of the symptoms varies, but is usually one or two weeks.

3.Clinical latency: The initial symptoms are followed by a stage called clinical latency, asymptomatic HIV, or chronic HIV..[2] Without treatment, this second stage of the natural history of HIV infection can last from about three years to over 20 years (on average, about eight years). While typically there are few or no symptoms at first, near the end of this stage many people experience fever, weight loss, gastrointestinal problems and muscle pains. ${ }^{[2]}$ Between 50 and $70 \%$ of people also develop persistent generalized lymphadenopathy, characterized by unexplained, non-painful enlargement of more than one group of lymph nodes (other than in the groin) for over three to six months. ${ }^{[3]}$ Although most HIV-1 infected http://escipub.com/american-journal-of-educational-research-and-reviews/ 
individuals have a detectable viral load and in the absence of treatment will eventually progress to AIDS, a small proportion (about $5 \%$ ) retain high levels of $\mathrm{CD} 4^{+} \mathrm{T}$ cells (T helper cells) without antiretroviral therapy for more than 5 years. These individuals are classified as HIV controllers or long-term nonprogressors (LTNP). Another group consists of those who maintain a low or undetectable viral load without anti-retroviral treatment, known as "elite controllers" or "elite suppressors". They represent approximately 1 in 300 infected persons.

\section{Acquired immunodeficiency syndrome:} Main symptoms of AIDS: Acquired immunodeficiency syndrome (AIDS) is defined in terms of either a CD4 ${ }^{+} \mathrm{T}$ cell count below 200 cells per $\mu \mathrm{L}$ or the occurrence of specific diseases in association with an HIV infection. In the absence of specific treatment, around half of people infected with HIV develop AIDS within ten years. The most common initial conditions that alert to the presence of AIDS are pneumocystis pneumonia $(40 \%)$, cachexia in the form of HIV wasting syndrome (20\%), and esophageal candidiasis. Other common signs include recurring respiratory tract infections. Opportunistic infections may be caused by bacteria, viruses, fungi, and parasites that are normally controlled by the immune system. Which infections occur depends partly on what organisms are common in the person's environment. These infections may affect nearly every organ system. People with AIDS have an increased risk of developing various viral-induced cancers, including Kaposi's sarcoma, Burkitt's lymphoma, primary central nervous system lymphoma, and cervical cancer. Kaposi's sarcoma is the most common cancer occurring in 10 to $20 \%$ of people with HIV. The second most common cancer is lymphoma, which is the cause of death of nearly $16 \%$ of people with AIDS and is the initial sign of AIDS in 3 to $4 \%$. Both these cancers are associated with human herpesvirus 8. Cervical cancer occurs more frequently in those with AIDS because of its association with human papillomavirus (HPV). Conjunctival cancer (of the layer that lines the inner part of eyelids and the white part of the eye) is also more common in those with HIV. Additionally, people with AIDS frequently have systemic symptoms such as prolonged fevers, sweats (particularly at night), swollen lymph nodes, chills, weakness, and unintended weight loss. Diarrhea is another common symptom, present in about $90 \%$ of people with AIDS. They can also be affected by diverse psychiatric and neurological symptoms independent of opportunistic infections and cancers.

\begin{tabular}{|c|c|}
\hline $\begin{array}{l}\text { 5. Transmission of HIV } \\
\text { Blood transfusion }\end{array}$ & $90 \%$ \\
\hline Childbirth (to child) & $25 \%$ \\
\hline Needle-sharing injection drug use & $0.67 \%$ \\
\hline Percutaneous needle stick & $0.30 \%$ \\
\hline Receptive anal intercourse ${ }^{*}$ & $0.04-3.0 \%$ \\
\hline Insertive anal intercourse ${ }^{*}$ & $0.03 \%$ \\
\hline $\begin{array}{l}\text { Receptive } \\
\text { intercourse }\end{array}$ & $\begin{array}{l}0.05- \\
0.30 \%\end{array}$ \\
\hline $\begin{array}{l}\text { Insertive } \\
\text { intercourse }\end{array}$ & $\begin{array}{l}0.01- \\
0.38 \%\end{array}$ \\
\hline Receptive oral intercourse ${ }^{*} \S$ & $0-0.04 \%$ \\
\hline Insertive oral intercourse ${ }^{\star} \S$ & $0-0.005 \%$ \\
\hline
\end{tabular}

HIV is transmitted by three main routes: sexual contact, significant exposure to infected body fluids or tissues, and from mother to child during pregnancy, delivery, or breastfeeding (known as vertical transmission). ${ }^{[10]}$ There is no risk of acquiring HIV if exposed to feces, nasal secretions, saliva, sputum, sweat, tears, urine, or vomit unless these are contaminated with blood. It is possible to be co-infected by more than one strain of HIV-a condition known as HIV superinfection.

6. Sexual: The most frequent mode of transmission of HIV is through sexual contact with an infected person. ${ }^{[10]}$ The majority of all 
Md. Abu Salek and Jannatun Nayeem, AJERR, 2018; 3:14

transmissions worldwide occur through heterosexual contacts (i.e. sexual contacts between people of the opposite sex); ${ }^{[10]}$ however, the pattern of transmission varies significantly among countries. In the United States, as of 2010, most transmission occurred in men who had sex with men, with this population accounting for $63 \%$ of all new cases. The risk from receiving oral sex has been described as "nearly nil"; however, a few cases have been reported. The per-act risk is estimated at $0-0.04 \%$ for receptive oral intercourse. In settings involving prostitution in low income countries, risk of female-to-male transmission has been estimated as $2.4 \%$ per act and male-to-female transmission as $0.05 \%$ per act. Risk of transmission increases in the presence of many sexually transmitted infections and genital ulcers. Genital ulcers appear to increase the risk approximately fivefold. Other sexually transmitted infections, such as gonorrhea, chlamydia, trichomoniasis, and bacterial vaginosis, are associated with somewhat smaller increases in risk of transmission. The viral load of an infected person is an important risk factor in both sexual and mother-to-child transmission. During the first 2.5 months of an HIV infection a person's infectiousness is twelve times higher due to this high viral load. If the person is in the late stages of infection, rates of transmission are approximately eightfold greater. Commercial sex workers (including those in pornography) have an increased rate of HIV. Rough sex can be a factor associated with an increased risk of transmission. Sexual assault is also believed to carry an increased risk of HIV transmission as condoms are rarely worn, physical trauma to the vagina or rectum is likely, and there may be a greater risk of concurrent sexually transmitted infections.

7. Body fluids: Blood-borne transmission can be through needle-sharing during intravenous drug use, needle stick injury, transfusion of contaminated blood or blood product, or medical injections with unsterilised equipment.
The risk from sharing a needle during drug injection is between 0.63 and $2.4 \%$ per act, with an average of $0.8 \%$. The risk of acquiring HIV from a needle stick from an HIV-infected person is estimated as $0.3 \%$ (about 1 in 333) per act and the risk following mucous membrane exposure to infected blood as $0.09 \%$ (about 1 in 1000) per act. In the United States intravenous drug users made up $12 \%$ of all new cases of HIV in 2009, and in some areas more than $80 \%$ of people who inject drugs are HIV positive. ${ }^{[10]} \mathrm{HIV}$ is transmitted in about $93 \%$ of blood transfusions using infected blood. In low income countries, only half of transfusions may be appropriately screened (as of 2008), and it is estimated that up to $15 \%$ of HIV infections in these areas come from transfusion of infected blood and blood products, representing between $5 \%$ and $10 \%$ of global infections. Although rare because of screening, it is possible to acquire HIV from organ and tissue transplantation. Unsafe medical injections play a significant role in HIV spread in sub-Saharan Africa. In 2007, between 12 and $17 \%$ of infections in this region were attributed to medical syringe use. The World Health Organization estimates the risk of transmission as a result of a medical injection in Africa at $1.2 \%$. Significant risks are also associated with invasive procedures, assisted delivery, and dental care in this area of the world. People giving or receiving tattoos, piercings, and scarification are theoretically at risk of infection but no confirmed cases have been documented. It is not possible for mosquitoes or other insects to transmit HIV.

8. Mother-to-child: HIV can be transmitted from mother to child during pregnancy, during delivery, or through breast milk resulting in infection in the baby. This is the third most common way in which HIV is transmitted globally. ${ }^{[10]}$ In the absence of treatment, the risk of transmission before or during birth is around $20 \%$ and in those who also breastfeed $35 \%$. As of 2008, vertical transmission accounted for about $90 \%$ of cases of HIV in children. With 
appropriate treatment the risk of mother-to-child infection can be reduced to about $1 \%$. Preventive treatment involves the mother taking antiretrovirals during pregnancy and delivery, an elective caesarean section, avoiding breastfeeding, and administering antiretroviral drugs to the newborn. Antiretrovirals when

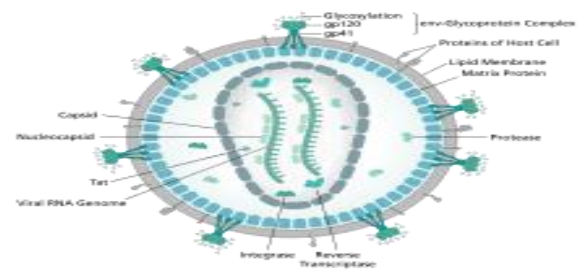

taken by either the mother or the infant decrease the risk of transmission in those who do breastfeed. Many of these measures are however not available in the developing world. If blood contaminates food during pre-chewing it may pose a risk of transmission.

\section{Virology on HIV}

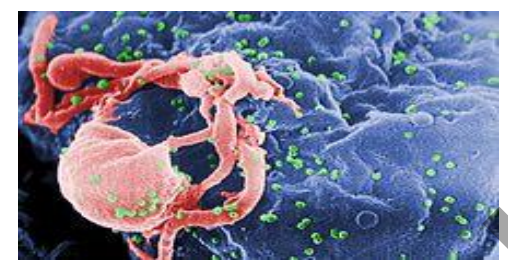

\section{Diagram of a HIV virion structure}

Scanning electron micrograph of HIV-1, colored green, budding from a cultured lymphocyte.

HIV is the cause of the spectrum of disease known as HIV/AIDS. HIV is a retrovirus that primarily infects components of the human immune system such as $\mathrm{CD}^{+}{ }^{+} \mathrm{T}$ cells, macrophages and dendritic cells. It directly and indirectly destroys $\mathrm{CD}^{+}{ }^{+} \mathrm{T}$ cells. HIV is a member of the genus Lentivirus, part of the family Retroviridae. Lentiviruses share many morphological and biological characteristics. Many species of mammals are infected by lentiviruses, which are characteristically responsible for long-duration illnesses with a long incubation period. Lentiviruses are transmitted as single-stranded, positive-sense, enveloped RNA viruses. Upon entry into the target cell, the viral RNA genome is converted (reverse transcribed) into double-stranded DNA by a virally encoded reverse transcriptase that is transported along with the viral genome in the virus particle. The resulting viral DNA is then imported into the cell nucleus and integrated into the cellular DNA by a virally encoded integrase and host co-factors. Once integrated, the virus may become latent, allowing the virus and its host cell to avoid detection by the immune system. HIV is now known to spread between CD4 ${ }^{+} \mathrm{T}$ cells by two parallel routes: cell-free spread and cell-to-cell spread, i.e. it employs hybrid spreading mechanisms. In the cell-free spread, virus particles bud from an infected T cell, enter the blood/extracellular fluid and then infect another T cell following a chance encounter. HIV can also disseminate by direct transmission from one cell to another by a process of cell-to-cell spread. The hybrid spreading mechanisms of HIV contribute to the virus's ongoing replication against antiretroviral therapies. Two types of HIV have been characterized: HIV-1 and HIV-2. HIV-1 is the virus that was originally discovered (and initially referred to also as LAV or HTLVIII). It is more virulent, more infective, and is the cause of the majority of HIV infections globally. The lower infectivity of HIV-2 as compared with HIV-1 implies that fewer people exposed to HIV-2 will be infected per exposure. Because of its relatively poor capacity for transmission, HIV-2 is largely confined to West Africa.

\section{Pathophysiology}

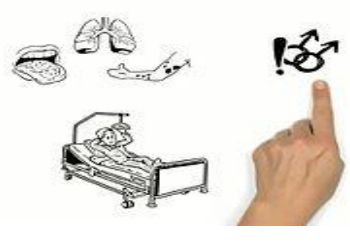

HIV/AIDS explained in a simple way

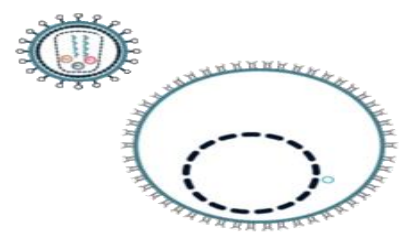

HIV replication cycle 
After the virus enters the body there is a period of rapid viral replication, leading to an abundance of virus in the peripheral blood. During primary infection, the level of HIV may reach several million virus particles per milliliter of blood. This response is accompanied by a marked drop in the number of circulating $\mathrm{CD} 4^{+}$ $T$ cells. The acute viremia is almost invariably associated with activation of $\mathrm{CD}^{+} \mathrm{T}$ cells, which kill HIV-infected cells, and subsequently with antibody production, or seroconversion. The $\mathrm{CD}^{+} \mathrm{T}$ cell response is thought to be important in controlling virus levels, which peak and then decline, as the $\mathrm{CD} 4^{+} \mathrm{T}$ cell counts recover. A good $\mathrm{CD} 8^{+} \mathrm{T}$ cell response has been linked to slower disease progression and a better prognosis, though it does not eliminate the virus. Ultimately, HIV causes AIDS by depleting $\mathrm{CD}^{+}{ }^{+} \mathrm{T}$ cells. This weakens the immune system and allows opportunistic infections. T cells are essential to the immune response and without them, the body cannot fight infections or kill cancerous cells. The mechanism of $\mathrm{CD}^{+} \mathrm{T}$ cell depletion differs in the acute and chronic phases. During the acute phase, HIV-induced cell lysis and killing of infected cells by cytotoxic $T$ cells accounts for $\mathrm{CD}^{+} \mathrm{T}$ cell depletion, although apoptosis may also be a factor. During the chronic phase, the consequences of generalized immune activation coupled with the gradual loss of the ability of the immune system to generate new $T$ cells appear to account for the slow decline in $\mathrm{CD}^{+} \mathrm{T}$ cell numbers. Although the symptoms of immune deficiency characteristic of AIDS do not appear for years after a person is infected, the bulk of $\mathrm{CD}^{+}{ }^{+} \mathrm{T}$ cell loss occurs during the first weeks of infection, especially in the intestinal mucosa, which harbors the majority of the lymphocytes found in the body. The reason for the preferential loss of mucosal CD4 ${ }^{+} \mathrm{T}$ cells is that the majority of mucosal $\mathrm{CD}^{+} \mathrm{T}$ cells express the CCR5 protein which HIV uses as a co-receptor to gain access to the cells, whereas only a small fraction of $\mathrm{CD} 4^{+} \mathrm{T}$ cells in the bloodstream do so. Immune activation, which is reflected by the increased activation state of immune cells and release of pro-inflammatory cytokines, results from the activity of several HIV gene products and the immune response to ongoing HIV replication. It is also linked to the breakdown of the immune surveillance system of the gastrointestinal mucosal barrier caused by the depletion of mucosal $\mathrm{CD}^{+} \mathrm{T}$ cells during the acute phase of disease.

11. HIV testing: Most people infected with HIV develop specific antibodies (i.e. seroconvert) within three to twelve weeks of the initial infection. Diagnosis of primary HIV before seroconversion is done by measuring HIV-RNA or p24 antigen. Positive results obtained by antibody or PCR testing are confirmed either by a different antibody or by PCR. Antibody tests in children younger than 18 months are typically inaccurate due to the continued presence of maternal antibodies. Thus HIV infection can only be diagnosed by PCR testing for HIV RNA or DNA, or via testing for the p24 antigen. Much of the world lacks access to reliable PCR testing and many places simply wait until either symptoms develop or the child is old enough for accurate antibody testing. In sub-Saharan Africa as of 2007-2009 between 30 and $70 \%$ of the population were aware of their HIV status. In 2009, between 3.6 and $42 \%$ of men and women in Sub-Saharan countries were tested. which represented a significant increase compared to previous years.

12.Classifications: Two main clinical staging systems are used to classify HIV and HIVrelated disease for surveillance purposes: the WHO disease staging system for HIV infection and disease, and the CDC classification system for HIV infection. Despite their differences, the two systems allow comparison for statistical purposes. The World Health Organization first proposed a definition for AIDS in 1986. Since then, the WHO classification has been updated and expanded several times, with the most recent version being published in 2007. The WHO system uses the following categories: 
- Primary HIV infection: May be either asymptomatic or associated with acute retroviral syndrome.

- Stage I: HIV infection is asymptomatic with a $\mathrm{CD} 4{ }^{+} \mathrm{T}$ cell count (also known as CD4 count) greater than 500 per microlitre $(\mu \mathrm{l}$ or cubic $\mathrm{mm}$ ) of blood. May include generalized lymph node enlargement.

- Stage II: Mild symptoms which may include minor mucocutaneous manifestations and recurrent upper respiratory tract infections. A CD4 count of less than $500 / \mu \mathrm{l}$.

- Stage III: Advanced symptoms which may include unexplained chronic diarrhea for longer than a month, severe bacterial infections including tuberculosis of the lung, and a CD4 count of less than 350/ $\mathrm{\mu l}$.

- Stage IV or AIDS: severe symptoms which include toxoplasmosis of the brain, candidiasis of the esophagus, trachea, bronchi or lungs and Kaposi's sarcoma. A CD4 count of less than 200/ $\mu$.

The United States Center for Disease Control and Prevention also created a classification system for HIV, and updated it in 2008 and 2014. This system classifies HIV infections based on CD4 count and clinical symptoms, and describes the infection in five groups. In those greater than six years of age it is:

- Stage 0: the time between a negative or indeterminate HIV test followed less than 180 days by a positive test

- Stage 1: CD4 count $\geq 500$ cells $/ \mu$ land no AIDS defining conditions

- Stage 2: CD4 count 200 to 500 cells/ $\mu \mathrm{l}$ and no AIDS defining conditions

- Stage 3: CD4 count $\leq 200$ cells $/ \mu$ or AIDS defining conditions

- Unknown: if insufficient information is available to make any of the above classifications
For surveillance purposes, the AIDS diagnosis still stands even if, after treatment, the $\mathrm{CD} 4^{+} \mathrm{T}$ cell count rises to above 200 per $\mu \mathrm{L}$ of blood or other AIDS-defining illnesses are cured. ${ }^{[3]}$

\section{Prevention of HIV/AIDS: Sexual contact:}

Consistent condom use reduces the risk of HIV transmission by approximately $80 \%$ over the long term. When condoms are used consistently by a couple in which one person is infected, the rate of HIV infection is less than $1 \%$ per year. There is some evidence to suggest that female condoms may provide an equivalent level of protection. Application of a vaginal gel containing tenofovir (a reverse transcriptase inhibitor) immediately before sex seems to reduce infection rates by approximately 40\% among African women. By contrast, use of the spermicide nonoxynol-9 may increase the risk of transmission due to its tendency to cause vaginal and rectal irritation. Circumcision in Sub-Saharan Africa "reduces the acquisition of HIV by heterosexual men by between $38 \%$ and $66 \%$ over 24 months".

14. Pre-exposure: Antiretroviral treatment among people with HIV whose CD4 count $\leq$ 550 cells/ $\mu \mathrm{L}$ is a very effective way to prevent HIV infection of their partner (a strategy known as treatment as prevention, or TASP). TASP is associated with a 10 to 20 fold reduction in transmission risk. Pre-exposure prophylaxis (PrEP) with a daily dose of the medications tenofovir, with or without emtricitabine, is effective in a number of groups including men who have sex with men, couples where one is HIV positive, and young heterosexuals in Africa. It may also be effective in intravenous drug users with a study finding a decrease in risk of 0.7 to 0.4 per 100 person years. Universal precautions within the health care environment are believed to be effective in decreasing the risk of HIV. Intravenous drug use is an important risk factor and harm reduction strategies such as needle-exchange programmes and opioid substitution therapy appear effective in decreasing this risk. 


\section{Mother-to-child: HIV and pregnancy:}

Programs to prevent the vertical transmission of HIV (from mothers to children) can reduce rates of transmission by $92-99 \%$. This primarily involves the use of a combination of antiviral medications during pregnancy and after birth in the infant and potentially includes bottle feeding rather than breastfeeding. If replacement feeding is acceptable, feasible, affordable, sustainable, and safe, mothers should avoid breastfeeding their infants; however exclusive breastfeeding is recommended during the first months of life if this is not the case. If exclusive breastfeeding is carried out, the provision of extended antiretroviral prophylaxis to the infant decreases the risk of transmission. In 2015, Cuba became the first country in the world to eradicate mother-to-child transmission of HIV.

16. Vaccination: HIV: Currently, there is no licensed vaccine for HIV or AIDS. ${ }^{[12]}$ The most effective vaccine trial to date, RV 144, was published in 2009 and found a partial reduction in the risk of transmission of roughly $30 \%$, stimulating some hope in the research community of developing a truly effective vaccine. Further trials of the RV 144 vaccine are ongoing.

16.1 Treatment: Management of HIV/AIDS: There is currently no cure or effective HIV vaccine. Treatment consists of highly active antiretroviral therapy (HAART) which slows progression of the disease. As of 2010 more than 6.6 million people were taking them in low and middle income countries. Treatment also includes preventive and active treatment of opportunistic infections. Disability-adjusted life year for HIV and AIDS per 100,000 inhabitants as of 2004.

$\begin{array}{ll}\text { no data } & 1000-2500 \\ \leq 10 & 2500-5000 \\ 10-25 & 5000-7500 \\ 25-50 & 7500-10000 \\ 50-100 & 10000-50000 \\ 100-500 & \geq 50000\end{array}$

500-1000

\section{Discrimination against people with HIV/AIDS}

AIDS stigma exists around the world in a variety of ways, including ostracism, rejection, discrimination and avoidance of HIV infected people; compulsory HIV testing without prior consent or protection of confidentiality; violence against HIV infected individuals or people who are perceived to be infected with HIV; and the quarantine of HIV infected individuals. Stigmarelated violence or the fear of violence prevents many people from seeking HIV testing, returning for their results, or securing treatment, possibly turning what could be a manageable chronic illness into a death sentence and perpetuating the spread of HIV.

\section{AIDS stigma has been further divided into the following three categories:}

- Instrumental AIDS stigma-a reflection of the fear and apprehension that are likely to be associated with any deadly and transmissible illness.

- Symbolic AIDS stigma-the use of HIV/AIDS to express attitudes toward the social groups or lifestyles perceived to be associated with the disease.

- Courtesy AIDS stigma-stigmatization of people connected to the issue of HIV/AIDS or HIV-positive people.

\section{Economic impact of HIV/AIDS and Cost of HIV treatment}

HIV/AIDS affects the economics of both individuals and countries. The gross domestic product of the most affected countries has decreased due to the lack of human capital. Without proper nutrition, health care and medicine, large numbers of people die from AIDS-related complications. They will not only be unable to work, but will also require significant medical care. Unemployment in people with HIV/AIDS also is associated with suicidal ideation, memory problems, and social isolation; employment increases self-esteem, 
sense of dignity, confidence, and quality of life. A 2015 Cochrane review found low-quality evidence that antiretroviral treatment helps people with HIV/AIDS work more, and increases the chance that a person with HIV/AIDS will be employed. By affecting mainly young adults, AIDS reduces the taxable population, in turn reducing the resources available for public expenditures such as education and health services not related to AIDS resulting in increasing pressure for the state's finances and slower growth of the economy. This causes a slower growth of the tax base, an effect that is reinforced if there are growing expenditures on treating the sick, training (to replace sick workers), sick pay and caring for AIDS orphans. This is especially true if the sharp increase in adult mortality shifts the responsibility and blame from the family to the government in caring for these orphans. At the household level, AIDS causes both loss of income and increased spending on healthcare. A study in Côte d'Ivoire showed that households having a person with HIV/AIDS spent twice as much on medical expenses as other households. This additional expenditure also leaves less income to spend on education and other personal or family investment.

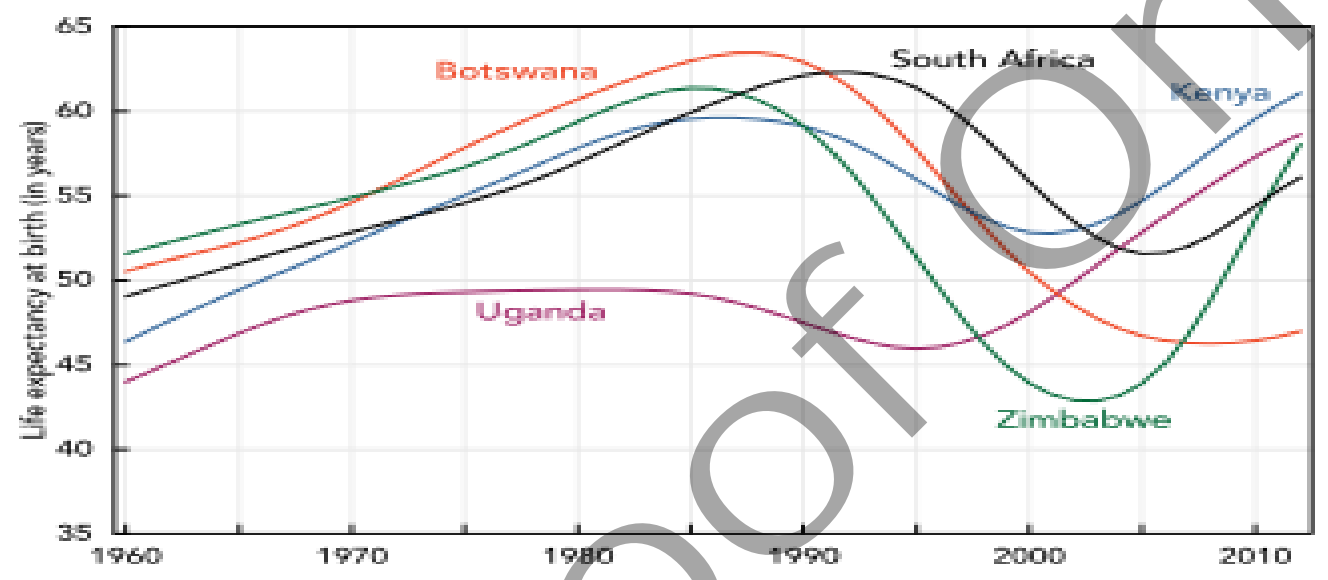

Changes in life expectancy in some African countries, 1960-2012

20. Criminal transmission of HIV: Criminal transmission of HIV is the intentional or reckless infection of a person with the human immunodeficiency virus (HIV). Some countries or jurisdictions, including some areas of the United States, have laws that criminalize HIV transmission or exposure. Others may charge the accused under laws enacted before the HIV pandemic.

21. HIV/AIDS research: HIV/AIDS research includes all medical research which attempts to prevent, treat, or cure HIV/AIDS along with fundamental research about the nature of HIV as an infectious agent and AIDS as the disease caused by HIV. Many governments and research institutions participate in HIV/AIDS research. This research includes behavioral health interventions such as sex education, and drug development, such as research into microbicides for sexually transmitted diseases, HIV vaccines, and antiretroviral drugs. Other medical research areas include the topics of pre-exposure prophylaxis, post-exposure prophylaxis, and circumcision and HIV.

\section{References:}

1. "World AIDS Day". World Health Organization. Retrieved June 16, 2015.

2. "Stages of HIV". U.S. Department of Health \& Human Services. Dec 2010. Retrieved June 13, 2012.

3. Mandell, Bennett, and Dolan (2010). Chapter 121.

4. "HIV Classification: CDC and WHO Staging Systems". Guide for HIV/AIDS Clinical Care. AIDS Education and Training Center Program. Retrieved November 21, 2015.

5. Sepkowitz KA (June 2001). "AIDS-the first 20 years". N. Engl. J. Med. 344 (23): 1764-72. doi:10.1056/NEJM200106073442306. PMID 11396444. 
6. editors, Alexander Krämer, Mirjam Kretzschmar, Klaus Krickeberg, (2010). Modern infectious disease epidemiology concepts, methods, mathematical models, and public health (OnlineAusg. ed.). New York: Springer. p. 88. ISBN 9780387938356.

7. Wilhelm Kirch (2008). Encyclopedia of public health. New York: Springer. pp. 676-677. ISBN 9781402056130.

8. "HIV/AIDS Fact sheet N³60". WHO. November 2015. Retrieved 11 February 2016.

9. "About HIV/AIDS". CDC. December 6, 2015. Retrieved 11 February 2016.

10. Markowitz, edited by William N. Rom ; associate editor, Steven B. (2007). Environmental and occupational medicine (4th ed.). Philadelphia: Wolters Kluwer/Lippincott Williams \& Wilkins. p. 745. ISBN 978-0-7817-6299-1. 